\title{
La reforma hacendaria y las transferencias en los municipios de Jalisco 2005-2011
}

\author{
The reform hacendaria and the transfers \\ in the municipalities of Jalisco 2005-2011
}

\author{
Antonio Ruíz-Porras* \\ Nancy García-Vázquez**
}

\begin{abstract}
Resumen
En el federalismo fiscal se definen los principios económicos, las fórmulas técnicas para la distribución de recursos, pero también los acuerdos políticos entre distintos niveles de gobierno. En más de una ocasión en el federalismo fiscal mexicano se han redefinido estos tres aspectos. La más reciente fue la reforma hacendaria del año 2007, denominada "Por los que menos tienen". Este nuevo arreglo tiene distintos comportamientos en las finanzas municipales que involucran criterios técnicos pero también políticos. Por ello, en este trabajo analizamos el sistema de transferencias en los 125 municipios de Jalisco. Particularmente, evaluamos cómo se comporta la asignación de transferencias federales y las participaciones estatales en la planeación de las finanzas municipales. $\mathrm{El}$ análisis contempla los ramos 28 y 33 y la suma de transferencias estatales y federales. Los resultados sugieren que la planeación en la asignación de las transferencias depende de factores poblacionales y territoriales como estableció la reforma, pero también obedece a factores políticos.
\end{abstract}

\section{Palabras clave:}

- Transferencias Intergubernamentales

- Planeación Gubernamental

- Municipios

\begin{abstract}
In the fiscal federalism there are defined the economic beginning, the technical formulae for the distribution of resources, but also the political agreements between different levels of government. In more than one occasion in the fiscal Mexican federalism these three aspects have been re-defined. The most recent was the reform hacendaria of the year 2007, named "For those who less have". This new arrangement has different behaviors in the municipal finance that involve technical criteria but also political. For it, in this work we analyze the system of transfers in 125 municipalities of Straw hat. Particularly, we evaluate how one endures the assignment of federal transfers and the state participations in the planeación of the municipal finance. The analysis contemplates the branches 28 and 33 and the sum of state and federal transfers. The results suggest that the Planning in the assignment of the transfers depends on population and territorial factors since the reform established, but also it obeys political factors.
\end{abstract}

\section{Keywords:}

- Intergovernmental transfers

- Government Planning

- Municipalities

JEL: H77, 021, D72

\section{Introducción}

En el federalismo fiscal, los distintos niveles de gobierno deben asumir de manera coordinada cómo han de distribuirse los recursos públicos. Se trata de un mecanismo para establecer los principios económicos, las fórmulas

*antoniop@cucea.udg.mx, Departamento de Métodos Cuantitativos, Universidad de Guadalajara-CUCEA, Zapopan, Jalisco, México.

** nancy.garcia@coljal.edu.mx, Dapartamento de Políticas Públicas Locales, Colegio de Jalisco, Jalisco, México. 
Economía Informa núm. 381 julio - agosto • 2013 ㅂ ㅁ |

técnicas para la distribución de recursos, pero también es una forma de acuerdo político. En más de una ocasión, en el federalismo fiscal mexicano se han redefinido estos tres aspectos. La más reciente fue la reforma hacendaria del año 2007, denominada "Por los que menos tienen", que tuvo como propósito disminuir la dependencia financiera las entidades, generando nuevos incentivos de recaudación y esfuerzo fiscal.

A casi seis años de esa reforma, poco se ha discutido su incidencia en la planeación y ejercicio de los recursos. ¿La reforma ha sido más equitativa y eficiente en la distribución de los recursos?, ¿está desprovista de consideraciones de tipo partidario?

En este trabajo analizamos el sistema de transferencias en los $125 \mathrm{mu}$ nicipios de Jalisco, antes y después de esta reforma justamente para observar si la planeación de los recursos públicos mantiene el espíritu de la iniciativa federal.

Como ya se ha dicho, en México las transferencias intergubernamentales son el principal recurso financiero de los municipios para atender las demandas de bienes públicos. Aunque en otros países las transferencias son un complemento en los ingresos locales, el Sistema Nacional de Coordinación Fiscal las ha convertido en la mayor fuente de financiamiento. ${ }^{1}$

Bajo este esquema, los municipios delegan la potestad del cobro de ciertos impuestos y aprovechamientos a cambio de recibir transferencias federales y participaciones estatales. Este acto de delegación tiene un efecto importante en la planeación presupuestal y en el establecimiento de políticas de desarrollo social y económico.

Los esquemas de transferencias federales y estatales han sido modificado con la reforma hacendaria de 2007. Por ejemplo, se reasignaron las transferencias, ya no sólo sobre la base de criterios territoriales, poblaciones o de progresividad, sino también del esfuerzo fiscal. Ahora la expectativa es que las entidades mantengan estos criterios e incentiven también a los gobiernos municipales a desarrollar un federalismo eficaz.

Una de las contribuciones de este análisis consiste en observar si se ha redefinido el sentido de la planeación de las finanzas locales. Aquí revisamos las tendencias de los criterios de la asignación presupuestal del gobierno de Jalisco hacia los 125 municipios. Para ello se analizan las relaciones entre las transferencias planeadas, la densidad poblacional y los partidos

${ }^{1}$ El Sistema Nacional de Coordinación Fiscal tiene su origen en las leyes Coordinación Fiscal (1978), del Impuesto al Valor Agregado (1978) y la Ley del Impuesto Especial sobre Producción y Servicios (1980). 
políticos, ello nos permite analizar los criterios técnicos y políticos (Krane y Wright 2000). Nuestras categorías de transferencias incluyen las participaciones estatales, las transferencias federales de los ramos 28 y 33 y la suma de éstas. El panel de datos incluye 873 observaciones anuales para el periodo 2005-2011. El análisis se realiza a través de gráficas de tendencia que proyectan los promedios de transferencias de grupos de municipios que comparten características comunes. Estos promedios se usan con fines comparativos y de análisis.

Los resultados principales de la investigación indican que la planeación de transferencias depende del grado de desarrollo y de los partidos políticos prevalecientes en los municipios. El análisis muestra que las transferencias crecieron entre 2005 y 2011 (medidas en pesos constantes per cápita); aunque no de manera constante. La evidencia también sugiere que las cantidades e incrementos en las transferencias dependen de los partidos.

Este estudio está dividido en seis secciones. En la primera se hace la presentación, en la segunda se revisa la literatura referida a las relaciones intergubernamentales y los principios normativos de las transferencias. En la tercera se analiza cómo está estructurado el sistema de transferencias y participaciones federales y estatales en su normatividad y administración a partir de la reforma hacendaria del 2007. En la cuarta se describen la base de datos, las variables y la metodología de análisis. En la quinta sección se presentan las tendencias de las transferencias planeadas, según la densidad poblacional y los partidos políticos. En la última sección se sintetizan y discuten los resultados.

\section{Revisión de la literatura}

Como refieren Rodden, Eskeland y Litvack, el federalismo fiscal es un mecanismo para intentar resolver problemas de gobernanza en la distribución de los bienes públicos, ${ }^{2}$ desde la perspectiva de un "planeador benevolente" (2003: 5).

La figura de un planeador centraliza ingresos y egresos mediante un diseño fiscal basado en las transferencias intergubernamentales como principal mecanismo de distribución. De modo que la habilidad de este planeador benevolente se refleja en el uso de las transferencias como mecanismo para:

\footnotetext{
${ }^{2}$ En la teoría del federalismo fiscal se argumenta que los gobiernos subnacionales y locales deben proveer bienes y servicios de manera eficiente con base en varias consideraciones. La primera se refiere a las ventajas de los gobiernos para conocer y satisfacer las preferencias de los habitantes de una localidad. La segunda se refiere a la potencial promoción de la competencia y eficiencia entre los productores de bienes y servicios locales. La tercera se refiere a las facilidades para monitorear y supervisar el manejo de los recursos públicos. Véase Hyman (2005) para una introducción a la teoría del federalismo fiscal.
} 
1) estabilización monetaria y fiscal, 2) redistribución del ingreso y la riqueza entre regiones y ciudadanos y 3) de asignación de bienes y servicios públicos y meritorios (Musgrave, 1959 y Oates, 1968). Se considera que la descentralización de estas funciones es necesaria para garantizar su eficiencia. Por esta razón, las funciones de estabilización y redistribución del ingreso suelen estar a cargo del gobierno federal; mientras que, la función de asignación y suministro de bienes y servicios suele estar a cargo de los gobiernos subnacionales o locales (Cabrera Castellanos y Lozano Cortés, 2011).

Este esquema de descentralización tiene dos implicaciones sobre la planeación financiera de los recursos. Por un lado, la capacidad de los gobiernos locales para redistribuir los recursos es limitada en tanto que debe observar los criterios generales del sistema de coordinación fiscal, y por otro, favorece una mayor focalización del gasto público, en tanto que los gobiernos locales tienen un vínculo más inmediato con respecto al demandante de servicios.

Para articular estos dos aspectos en un único mecanismo, el sistema de transferencias contempla aquellas que son condicionadas, es decir, que se ajustan a criterios de asignación basados en necesidades prioritarias como la salud o la educación; y aquellas que son no condicionadas, que son de compensación y reducción de la brecha fiscal (Boadway y Shah, 2009: 255).

Cuando un país define su sistema de transferencias, está discutiendo simultáneamente un asunto técnico y político. Es técnico en tanto que se deben establecer los criterios económicos y las fórmulas para la distribución de los recursos financieros. Pero también es un asunto político en tanto que actores o partidos políticos buscarán obtener un beneficio político o electoral. ${ }^{3}$

Paradójicamente, y pese a su importancia, son relativamente escasos los estudios que han analizado el sistema de planeación de las transferencias en México a nivel municipal. Esta carencia de estudios es sorprendente si se consideran las desigualdades al interior del país y la descentralización fiscal en que ha estado envuelta la economía mexicana desde 1980. Algunos trabajos analizan algunos de los fondos transferidos (Hernández y Jarillo, 2007) o los incentivos fiscales (Sour 2004 y 2008). ${ }^{4}$ Pero la planeación no

\footnotetext{
${ }^{3}$ La asignación política de los recursos en el federalismo mexicano, así como los límites políticos de la redistribución del gobierno ha sido estudiados y discutidos desde distintas perspectivas. Véase, entre otros, los estudios de Díaz Cayeros (1997) y (2004). Una línea de investigación es el llamado "efecto matamoscas" (flypaper effect), planteado originalmente por Bradford y Oates (1971). Véase los estudios de Sour y Girón (2009) y Cárdenas y Sharma (2011) para la evolución del mismo en el contexto de los municipios mexicanos.

${ }^{4}$ Otros trabajos son: Kraemer, 1997; Díaz Cayeros, Alberto, (1997, 2004); Hernández Trillo, Fausto y Brenda Jarillo Rabling, (2007), Ibarra Salazar, 2003; Cabrera Castellanos y Lozano Cortés ( 2011); aunque no estudian todos los ramos que componen las transferencias ni su proceso de planeación.
} 
parecería ser un tema relevante. Por esta razón, aquí se considera que debieran revisarse con mayor cuidado.

\section{Los sistemas de transferencias federales y estatales en Jalisco}

En este apartado exponemos los mecanismos del los sistemas de transferencias federales y estatales y su proceso de planeación. En esta parte nos enfocamos en algunos de los aspectos técnicos de la planeación de las transferencias federales y estatales en Jalisco. En primer lugar hay que tomar en cuenta el marco federal y, posteriormente, revisar cómo éste baja al nivel de los municipios jaliscienses.

Los mecanismos de asignación de las transferencias federales

Desde 1980, la Recaudación Federal Participable (RFP) es el instrumento administrativo-financiero para determinar la distribución de los recursos del gobierno central hacia los gobiernos subnacionales. ${ }^{5}$ La reforma de 2007 modificó la RFP y parte de las fórmulas de las participaciones y las aportaciones. Las leyes modificadas fueron la de Coordinación Fiscal (LCF), del Impuesto Especial sobre Producción y Servicios (LIEPS) y de Derechos (LFD).

Por lo que se refiere al Ramo 28 referente a las Participaciones a Entidades Federativas y Municipios (transferencias no condicionadas), por ley, al menos 20\% debe destinarse a los municipios. Su distribución se basa en la población, la capacidad recaudatoria de las entidades federativas y, aspectos específicos de acuerdo al fondo. ${ }^{6}$

El Ramo 33 relativo a las Aportaciones a Entidades Federativas y Municipios (transferencias condicionadas), comprende la mayor cantidad de recursos, pero están sujetos a la ejecución de políticas sociales. ${ }^{7} \mathrm{Su}$ distribución depende de la RFP y de los índices de pobreza.

\footnotetext{
${ }^{5}$ La RFP adiciona todos los impuestos recaudados por la federación (ingresos tributarios), más los derechos sobre la extracción de petróleo y la minería (restando las devoluciones por tales conceptos). La RFP no considera derechos adicionales o extraordinarios ni impuestos o servicios convenidos entre la federación y las entidades.

${ }^{6}$ Una mayor descripción de los criterios de asignación puede consultarse en la Secretaría de Hacienda y Crédito Público, en su portal http://www.transparenciapresupuestaria.gob.mx

${ }^{7}$ El Ramo 33 es una derivación de otros ramos, programas y recursos que se denominaban 12 , 25 y 26. Mayor detalle de estos fondos puede encontrarse en el boletín del Centro de Estudios de las Finanzas Públicas http://www.cefp.gob.mx/intr/edocumentos/pdf/cefp/cefp0362006. pdf (Consultado el 6/10/12).
} 


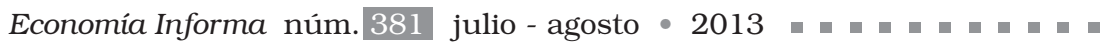

Los mecanismos para transferencias federales en Jalisco

En Jalisco, la planeación de las transferencias compete a los ejecutivos estatal y municipal, así como al congreso local. Este proceso está regulado por distintas leyes. ${ }^{8}$ La principal es la Ley de Coordinación Fiscal del Estado de Jalisco con sus municipios. De un modo general, hay una serie de criterios básicos en este documento.

1. El gobierno estatal asigna hasta $22 \%$ de la RFP a los municipios

2. Todas las participaciones (federales y estatales) se reúnen en el Fondo Municipal de Participaciones

3. La garantía es que ningún municipio recibirá una transferencia menor al año anterior

4. Se considera la recaudación de los ingresos propios del municipio y sus índices de marginación y de población

5. Las participaciones del gobierno estatal se obtienen del cobro de los derechos estatales.

Con base en la información poblacional del Instituto Nacional de Estadística y Geografía (INEGI) se estiman la proporción del número de habitantes de cada municipio en relación al total estatal, así como el índice de marginalidad. Asimismo, se reparten las participaciones y aportaciones federales sobre la base del esfuerzo recaudatorio local. Los fondos se asignan a partir de un coeficiente que se integra; primero, por 50\% de las participaciones federales y los ingresos estatales; segundo, por la clasificación de todos los municipios considerando el grado de marginalidad. ${ }^{9}$

Del Ramo 28, se integra el Fondo Municipal de Particiones (FMP) que es el instrumento administrativo-financiero para adicionar tanto los recursos estatales como los federales. Para su asignación, el FMP toma como referentes las siguientes proporciones (gráfica 1).

${ }^{8}$ Constitución Política del Estado de Jalisco; Ley Orgánica del Poder Ejecutivo del Estado de Jalisco; Ley de Fiscalización Superior del Estado de Jalisco; Ley de Planeación para el Estado de Jalisco y sus Municipios; Ley del Presupuesto, Contabilidad y Gasto Público del Estado; Ley de Transparencia e Información Pública del Estado de Jalisco; Ley de Obra Pública del Estado de Jalisco; Ley General de Contabilidad Gubernamental; Lineamientos Generales para la Gestión Financiera de la Inversión Pública; Manual de Normas y lineamientos presupuestales; Clasificador por Objeto del Gasto.

${ }_{9}^{9}$ Además, los municipios también reciben otros recursos. Como parte del Ramo 39 denominado Programa de Apoyos al Fortalecimiento de las Entidades Federativas (PAFEF), reciben el Fondo de Incentivo a la Eficiencia en la Gestión Municipal (FIEGM) y el Fondo de Estabilización de los Ingresos de las Entidades Federativas. Estos fondos se integran con el uno por ciento de los ingresos del estado respectivamente. 
Por lo que se refiere al Ramo 33, la Secretaría de Finanzas debe entregar lo correspondiente al Fondo de Fortalecimiento y al Fondo de Infraestructura, apegándose a las reglas establecidas por la federación dado que se trata de transferencias condicionadas.

\section{Gráfica 1}

\section{Criterios de distribución del Fondo Municipal de Participaciones}

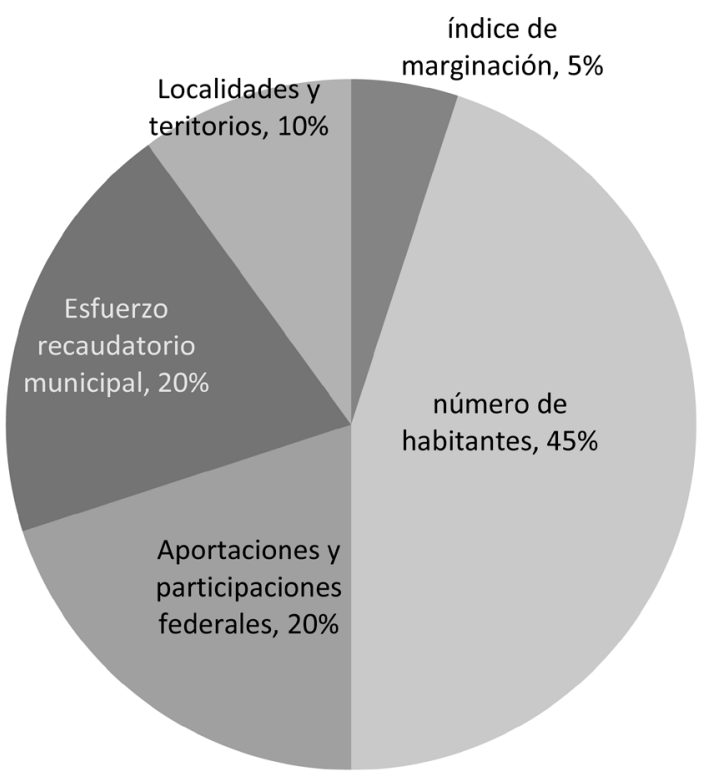

Fuente: elaboración propia.

\section{Base de datos y metodología de análisis}

Nuestro análisis se realiza con datos de los procesos estatales de planeación gubernamental a nivel municipal, la densidad poblacional y la composición política de los ayuntamientos. Son cifras anuales estimadas por el gobierno del estado ${ }^{10}$ que se publican en el primer trimestre. ${ }^{11}$

\footnotetext{
${ }^{10}$ Las transferencias planeadas son presupuestadas y publicadas por el gobierno de Jalisco. Las mismas pueden consultarse en http://www.jalisco.gob.mx (consultado el 12/10/2011).

${ }^{11}$ Particularmente, las cifras de participaciones del periodo 2005-2008 son obtenidas de los marcos programático-presupuestales del gobierno estatal. Las cifras del periodo 2009-2011 son obtenidas de la Secretaria de Finanzas estatal. Todas las cifras anuales están expresadas en pesos constantes per cápita. Los índices del precios usados para deflactar las transferencias estatales y federales son los correspondientes al INPC de la segunda quincena de junio de cada año. La base de dicho índice corresponde a la segunda quincena de diciembre 2010.
} 
Las transferencias las dividimos en cuatro categorias. Una que incluye las participaciones estatales (impuesto de $2 \%$ sobre nómina y $2 \%$ sobre hospedaje). Otra incluye las participaciones federales del Ramo 28. Una más contiene las aportaciones federales del Ramo 33. Finalmente, la última agrega la suma de las transferencias estatales y federales.

Como ya referimos, un criterio técnico de las transferencias es el grado de desarrollo y población, aquí se emplea como proxy el indicador de densidad poblacional (dens). ${ }^{12}$ Así se dividen los municipios en cinco grupos (medidos en hab $/ \mathrm{km}^{2}$ ): Muy baja $(0<$ dens $<10)$, baja $(10 \leq$ dens $<50$ ), media $(50 \leq$ dens $<100)$, alta $(100 \leq$ dens $<1000)$ y muy alta (dens $\geq 1000)$. Esta clasificación es la propuesta por Flamand (2007). Nuestra variable de contraste es de naturaleza política. Se basa en los resultados del Instituto Electoral y de Participación Ciudadana (IEPC) del estado de Jalisco para la elección a presidente municipal (comicios de 2003, 2006 y 2009). La preferencia política en cada municipio es la del partido postulante del presidente electo. ${ }^{13}$ Aquí se consideran tres alternativas a nivel: PAN, PRI y otros partidos políticos.

En relación a la planeación de las transferencias, para cada año se calculan los promedios de las cantidades asignadas para cada categoría de transferencia. Estos promedios se estiman para municipios que comparten grados de desarrollo y de preferencia comunes.

\section{Análisis de tendencias}

En esta sección se muestran mediante gráficas cómo se dan las transferencias planeadas, la densidad poblacional y los partidos políticos. Las gráficas proyectan un análisis descriptivo basado en el promedio de las transferencias per cápita según los grupos de densidad poblacional o los tres grupos de municipios para el periodo 2005-2011.

La gráfica 2 que agrupa la suma de todas las aportaciones y participaciones muestra un importante crecimiento a partir de 2008, un año después de la reforma y es cuando alcanzan un nivel máximo. También se observa que el total de transferencias estimadas está inversamente relacionado con

\footnotetext{
${ }^{12}$ Este supuesto se sustenta en los hallazgos de Martínez Pellegrini, Flamand y Hernández (2008). En este estudio se muestra que, en los municipios mexicanos, una mayor densidad poblacional se asocia a un menor grado de marginalización y subdesarrollo.

${ }^{13}$ En el Apéndice (cuadro 2.A), se muestra cómo los partidos políticos ocuparon las presidencias municipales y, por tanto, la preferencia política a nivel municipal en el periodo 2005-2011.
} 
el grado de desarrollo de los municipios. Este hecho es interesante porque indica que, en el agregado, en la planeación predomina un criterio redistributivo progresivo. Incluso en 2011, el promedio de transferencias per cápita para los municipios de muy baja densidad resulta mayor.

\section{Gráfica 2}

\section{Transferencias estimadas y densidad poblacional}

a nivel municipal en Jalisco, 2005-2011

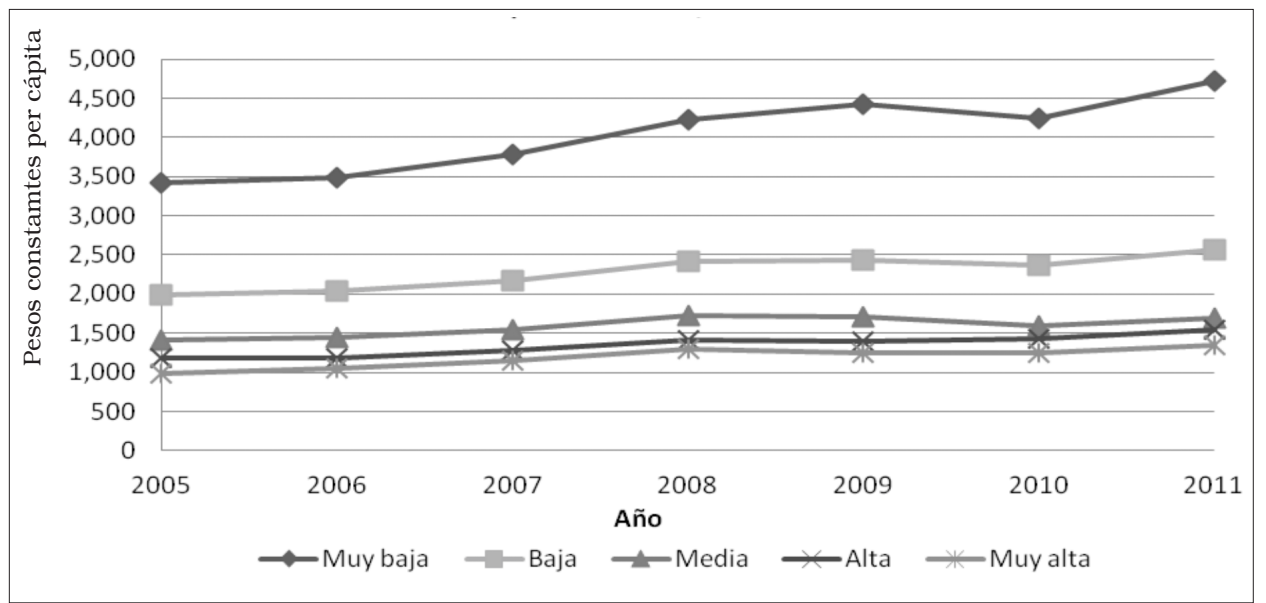

Fuente: elaboración propia con base en estimaciones del gobierno del Estado de Jalisco. Nota: la taxonomia usada para clasificar a los municipios sigue a Flamand (2007). Las cifras están redondeadas y expresadas en pesos de diciembre de 2010.

De modo que esta primera gráfica muestra que sí se están cumpliendo los principios de compensación y redistribución, aunque los datos no permiten evaluar si aumentó el esfuerzo fiscal, sí reflejan que el gobierno jalisciense planeó con un mayor pronóstico de gasto los presupuestos de 2008 y 2009, pero luego hubo una contracción del gasto derivada quizá de la crisis financiera mundial. Consistentemente, la segunda gráfica también reporta un incremento en las transferencias recibidas por todos los partidos políticos. Sin embargo, se evidencia la existencia de criterios políticos en tanto que, comparativamente, hay diferencias significativas.

Particularmente entre 2005 y 2009, las transferencias hacia los municipios del PRI son más altas que aquellas de los municipios del PAN. Sólo en 2010 esta situación se revierte a favor del PAN. Las menores transferencias son para municipios donde prevalecen otros partidos. 


\section{Gráfica 3 \\ Transferencias estimadas (federales y estatales) y partidos políticos a nivel municipal en Jalisco, 2005-2011}

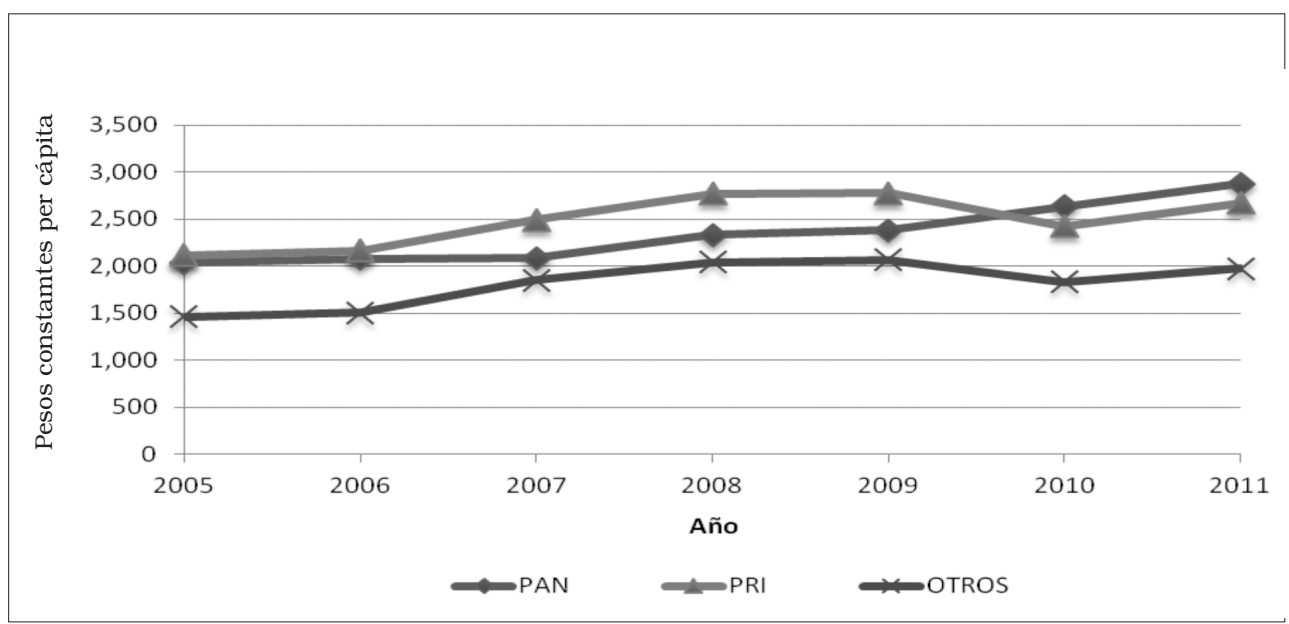

Fuente: elaboración propia con base en estimaciones del gobierno del Estado de Jalisco. La taxonomia usada para clasificar a los municipios sigue a Flamand (2007). Las cifras están redondeadas y expresadas en pesos de diciembre de 2010.

\section{Conclusiones y discusión}

En esta investigación hemos estudiado el sistema de transferencias en los 125 municipios jaliscienses. Hemos analizado las relaciones entre las transferencias planeadas, la densidad poblacional y los partidos políticos. Los resultados del análisis sugieren que la planeación de las transferencias hacia los municipios depende del grado de desarrollo y de los partidos políticos. Particularmente, las transferencias per cápita medias destinadas a los de baja y muy baja densidad y a municipios del PRI y del PAN son mayores que la media del total de municipios. También se observa que las transferencias crecieron entre 2008 y 2011 (medidas en pesos constantes per cápita); aunque no de manera constante. La evidencia también sugiere que las cantidades e incrementos en las transferencias dependen de los partidos.

Los resultados de esta investigación tienen implicaciones para evaluar las relaciones intergubernamentales y el sistema de transferencias en los municipios de Jalisco. Una implicación es que la planeación gubernamental está limitada para generar bienes y servicios de manera adecuada. Esta 
afirmación se sustenta en el análisis teórico de Bradford y Oates (1971) sobre los efectos de las transferencias en la producción de bienes y servicios públicos locales. Como es conocido, ellos muestran que, en la presencia de equilibrios políticos, hay menores niveles de eficiencia e impacto de las transferencias cuando éstas son no condicionadas.

Finalmente, creemos necesario extender el análisis desarrollado a fin de abarcar la implementación, la ejecución, sus determinantes y la correspondencia entre etapas. En este contexto, y al considerar la existencia de discriminación política, el lobbying quizá debiera ocupar un lugar relevante en estudios posteriores. Al final de este trabajo, se evidencia que analizar la planeación presupuestaria desde los aspectos técnicos y políticos nos permite observar un panorama más claro de porqué se sostienen condiciones sumamente diversas entre los gobiernos locales.

\section{Bibliografía}

Bradford, David F. y Wallace E. Oates, "Towards a predictive theory of intergovernmental grants”, American Economic Review, 1971, 61(2), mayo, pp. 440-448.

Boadway, Robin y Anwar Shah, Fiscal Federalism Principles and Practices of Multiorder Governance, Canada, Queens University, 2008.

Cabrera Castellanos, Fernando y René Lozano Cortés, Relaciones Intergubernamentales y el Sistema de Transferencias en México: una propuesta de nivelación interjurisdiccional, México, Universidad de Quintana Roo y Miguel Ángel Porrúa, 2011.

Cárdenas, Oscar J. y Amarendra Sharma, "Mexican municipalities and the flypaper effect”, Public Budgeting and Finance, 31(3), Otoño, pp. 73-93, 2011.

Díaz Cayeros, Alberto, “Asignación política de recursos en el federalismo mexicano: Incentivos y limitaciones”, Perfiles Latinoamericanos, 1997, 6(10), junio, pp. 35-73.

Díaz Cayeros, Alberto, "El federalismo y los límites políticos de la redistribución”, Gestión y Politica Pública, 2004 13(3), segundo semestre, pp. 663-687.

Flamand, Laura (responsable técnico), Índice de Desarrollo Municipal Básico 2000 El Colegio de la Frontera Norte, Tijuana, 2007. 
Hernández Trillo, Fausto y Brenda Jarillo Rabling, "Transferencias condicionadas federales en países en desarrollo: el caso del FISM en México", Estudios Económicos, 2007, 22(2), julio-diciembre, pp. 143-184.

Hyman, David N., Public Finance: A Contemporary Application of Theory to Policy, Octava edición, Thomson South Western, Mason, 2005.

Ibarra Salazar, Jorge, "Dependencia financiera en las participaciones federales de los estados fronterizos mexicanos”, Frontera Norte, 2003, 15(29), enero-junio, pp. 87-123.

Kraemer, Moritz, "Intergovernmental transfers and political representation: Empirical evidence from Argentina, Brazil and Mexico", Working Paper 345, Inter-American Development Bank, Washington D.C, 1997.

Krane, Dale y Deil S. Wright, “Intergovernmental relations”, en Jay M. Shafritz (ed.), Defining Public Administration: Selections from the International Encyclopedia of Public Policy and Administration, Westview Press, Boulder, 2000, pp. 83-101.

Musgrave, Richard A., The Theory of Public Finance: A Study in Public Economy, McGraw-Hill, Nueva York, 1959.

Oates, Wallace E., "The theory of public finance in a federal system", The Canadian Journal of Economics, 1968, 1(1), febrero, pp. 37-54.

Sour, Laura, "El sistema de transferencias federales en México: ¿Premio o castigo para el esfuerzo fiscal de los gobiernos locales urbanos?”, Gestión y Política Pública, 2004, 13(3), pp. 733-751.

Sour, Laura, "Un repaso sobre los conceptos de sobre capacidad y esfuerzo fiscal, y su aplicación en los gobiernos locales mexicanos”, Estudios Demográficos y Urbanos, 2008, 23(2), pp. 271-297.

Sour Laura y Fredy Girón, "Electoral competition and the flypaper effect in Mexican local governments", Documento de Trabajo 238, México, CIDE, 2009. 